\title{
BEM formulation based on dipoles of stresses applied to crack growth modelling in quasi-brittle materials
}

\author{
H. L. Oliveira \& E. D. Leonel \\ Department of Structural Engineering, School of Engineering of \\ São Carlos, University of São Paulo, Brazil
}

\begin{abstract}
Fracture mechanics and crack propagation problems have been widely studied by the scientific community in recent years, because crack growth phenomenon can explain the failure of structures. In order to model accurately the structural behaviour of complex engineering structures, including complex geometries and boundary conditions, numerical techniques are required. In this regard, the boundary element method (BEM) has been widely used to solve complex engineering problems, especially those where its mesh dimension reduction includes advantages on the modelling. This paper addresses the analysis of crack propagation in quasi-brittle materials using an alternative BEM formulation. In this type of problem, the damaged zone ahead of the crack tip is modelled based on the fictitious crack model. Therefore, the residual resistance of the damaged zone is represented by cohesive stresses, which tends to close the crack faces. The alternative BEM formulation proposed aims at modelling the cohesive stresses using the domain term of the direct integral representation. This term is modified, in order to be non null only at the fictitious crack path. As a result of the domain term manipulation, appears a dipole of stresses, which will govern the cohesive stresses. It leads a nonlinear formulation, where the cohesive stresses are determined according the crack opening displacement. In this paper, the nonlinear problem is solved using a tangent operator, which includes the nonlinear laws into the algebraic BEM equations. This operator is derived considering linear, bi-linear and exponential cohesive laws. The results achieved by the proposed formulation are compared with experimental and numerical results in order to validate and prove its robustness and accuracy. Keywords: tangent operator, dipoles of stresses, cohesive crack modelling.
\end{abstract}




\section{Introduction}

The linear elastic fracture mechanics is correctly applied when the size of the fracture process zone (FPZ) in front of the crack tip is small compared to the size of the crack and the size of the specimen, as observed in brittle bodies. When quasi-brittle materials are considered, other models must be used to take into account the behaviour of the FPZ. The cohesive crack model is the simplest of such nonlinear fracture mechanics models. Considering the cohesive crack model, the propagation is governed by a traction-displacement relation across the crack faces near the tip. This model was introduced in the early sixties for ductile materials by Barenblatt [1] and Dugdale [2]. In the late seventies, Hillerborg et al. [3] introduced the concept of fracture energy into the cohesive crack model and proposed a number of traction-displacement relationships for concrete.

Cohesive crack model has been included in several numerical methods as boundary element method (BEM), mesh less methods and finite element method (FEM). Several algorithms have been proposed in order to solve the nonlinearity coming from the FPZ [4]. When cohesive crack growth is simulated, one of the most significant difficulties that arise is the requirement of mesh's conformity to the geometry of the crack. It needs remeshing procedures during crack propagation, leading a high computational cost for large and complexes geometries and boundary conditions. In this regard, FEM is not a numerical technique completely adapted to solve this problem. Due to its domain mesh, the remeshing procedure is not a simple task. Moreover, FEM requires a very fine mesh in order to represent the stress singularity at the crack tip, leading the construction of large stiffness' matrix and, consequently, low performance. Despite these difficulties, there are some works in literature associating the cohesive crack growth problem to FEM, as presented by [5].

On the other hand, BEM has been extensively used to solve many problems in engineering, especially those related to fracture mechanics. In such problems, this numerical method requires only boundary and crack surface discretizations. Then, remeshing difficulties are considerable avoided. Moreover, compared to domain mesh approaches, BEM is more efficient, particularly in solving mixed mode crack propagation problems, due to its efficiency in stress concentration modelling and mesh reduction aspects $[6,7]$.

$\mathrm{BEM}$ has been used by many researchers in order to handle cohesive crack growth modelling. Among them, it is worth to mention [8], where the dual BEM formulation was used to simulate cohesive mixed mode crack growth in plane structures composed by concrete. [9] used the single domain dual boundary element formulation as a boundary element approach. In this formulation, the cohesive zone is incorporated into the formulation, resulting in a nonlinear problem. The multi-domain BEM and the symmetric Galerkin BEM were used by [10] and [11], respectively, for analyses of cohesive crack growth.

During decades, numerical methods have been used and regarded as suitable tools to predict the fracture and failure of engineering structures, leading the creation of a science denominated computational fracture mechanics. Due to the large advances in the field of science and engineering, the need to analyse larger 
and more complex structures in numerical form has become a challenger issue. Therefore, the development of numerical tools and more efficient algorithms regarding the computational cost and accuracy of results is quite essential. In this context, this paper presents an alternative BEM formulation based on dipoles of stresses applied to model cohesive crack propagation. The proposed formulation aims at modelling the cohesive stresses, on the fictitious crack, using the domain term of the direct BEM integral representation. This term is modified, in order to be non null only at the fictitious crack path. As a result of the domain term manipulation, appears a dipole of stresses, which will govern the cohesive stresses. This formulation solves the crack growth problem using three algebraic equations per source point positioned at the crack path. By comparing it with the classical dual BEM, that uses four algebraic equations per crack source point, it is a great advantage.

The proposed formulation is nonlinear, since the cohesive stresses are determined according the crack opening displacement values. In this work, the nonlinear problem is solved using a tangent operator. This kind of operator includes the nonlinear laws into the algebraic BEM equations, leading a faster and accurate solution. The tangent operator was derived for linear, bi-linear and exponential cohesive laws, which are the main contribution of this paper. In order to compare the performance of the tangent operator, the nonlinear problem was also solved by the classical approach, in which the corrections into the cohesive crack stresses are performed by applying a non-equilibrated stress vector and keeping constant all relevant matrices. The results achieved by the proposed formulation are compared with experimental and numerical ones, in order to validate and prove its robustness and accuracy.

\section{BEM integral equations}

Considering an elastic body with domain $\Omega$ and boundary $\Gamma$ subjected to an initial stress field $\sigma_{j k}^{0}$ acting into the domain, the following integral representation can be written, as presented by [12]

$$
c_{l k}^{i} u_{k}^{i}+\int_{\Gamma} p_{l k}^{*} u_{k} d \Gamma=\int_{\Gamma} u_{l k}^{*} p_{k} d \Gamma+\int_{\Omega_{0}} \sigma_{j k}^{0} \varepsilon_{l j k}^{*} d \Omega_{0}
$$

where $u_{l k}^{*}, p_{l k}^{*}$ and $\varepsilon_{l k j}^{*}$ are the well known fundamental solutions for displacements, tractions and strains, respectively [12]. $\Omega_{0}$ is the initial stress region, which for the problem studied represents the FPZ. The initial stress region is a wide thin region limited by a boundary $\bar{\Gamma}^{C}$, where $\bar{\Gamma}^{C}=\bar{\Gamma}_{1}^{C} \cup \bar{\Gamma}_{2}^{C}$ as presented in Fig. 1.

In order to develop the cohesive fracture formulation, the initial stress integral shown in Eq. (1) has to be conveniently manipulated. Initially, it can be integrated over the initial stress region leading to

$$
\int_{\Omega_{0}} \sigma_{j k}^{0} \varepsilon_{l j k}^{*} d \Omega_{0}=\int_{\bar{\Gamma}^{C}} u_{l j}^{*} \sigma_{j k}^{0} \eta_{k} d \bar{\Gamma}^{C}-\int_{\Omega_{0}} u_{l j}^{*} \sigma_{j k, k}^{0} d \Omega_{0}
$$




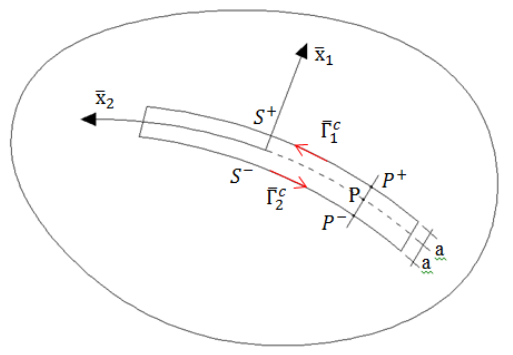

Figure 1: Initial stress region.

in which $\eta_{k}$ is the normal vector to boundary $\bar{\Gamma}^{C}$ and the term $\sigma_{j k}^{0} \eta_{k}$ represents the tractions at the initial stress region boundaries, $p_{j}^{01}$. Therefore, the first integral on right hand side of Eq. (2) can be rewritten as

$$
\int_{\bar{\Gamma}^{C}} u_{l j}^{*}(f, S) p_{j}^{01}(S) d \bar{\Gamma}^{C}=\int_{\bar{\Gamma}_{1}^{C}} u_{l j}^{*}\left(f, S^{+}\right) p_{j}^{01}\left(S^{+}\right) d \bar{\Gamma}_{1}^{C}+\int_{\bar{\Gamma}_{2}^{C}} u_{l j}^{*}\left(f, S^{-}\right) p_{j}^{01}\left(S^{-}\right) d \bar{\Gamma}_{2}^{C}
$$

where $S^{+}$and $S^{-}$are field points positioned at boundaries $\bar{\Gamma}_{1}^{C}$ and $\bar{\Gamma}_{2}^{C}$, respectively.

Assuming that the width of initial stress region is too small compared to its length; then, the kernels defined at $S^{+}$and $S^{-}$can be rewritten for the middle path of the region $S$. Therefore, these kernels can be redefined using Taylor series expansion as follows

$$
\begin{aligned}
& u_{l j}^{*}\left(f, S^{+}\right)=u_{l j}^{*}(f, S)+\frac{\partial u_{l j}^{*}(f, S)}{\partial \bar{x}_{1}} a \\
& u_{l j}^{*}\left(f, S^{-}\right)=u_{l j}^{*}(f, S)-\frac{\partial u_{l j}^{*}(f, S)}{\partial \bar{x}_{1}} a
\end{aligned}
$$

Considering the expansion terms presented by Eq. (4), it can be used to rewrite Eq. (3) as

$$
\begin{aligned}
& \int_{\bar{\Gamma}^{C}} u_{l j}^{*}(f, S) p_{j}^{01}(S) d \bar{\Gamma}^{C}= \\
& \int_{\bar{\Gamma}_{1}^{C}}\left[u_{l j}^{*}(f, S)+\frac{\partial u_{l j}^{*}(f, S)}{\partial \bar{x}_{1}} a\right] p_{j}^{01}\left(S^{+}\right) d \bar{\Gamma}_{1}^{C}+\int_{\bar{\Gamma}_{2}^{C}}\left[u_{l j}^{*}(f, S)-\frac{\partial u_{l j}^{*}(f, S)}{\partial \bar{x}_{1}} a\right] p_{j}^{01}\left(S^{-}\right) d \bar{\Gamma}_{2}^{C}
\end{aligned}
$$

Assuming that $d \bar{\Gamma}_{1}^{C}=d \bar{\Gamma}$ and $d \bar{\Gamma}_{2}^{C}=-d \bar{\Gamma}$, the equation above can be rewritten as

$$
\int_{\bar{\Gamma}^{C}} u_{l j}^{*}(f, S) p_{j}^{01}(S) d \bar{\Gamma}^{C}=\int_{\bar{\Gamma}} \frac{\partial u_{l j}^{*}(f, S)}{\partial \bar{x}_{1}} p_{j}^{01}(S) 2 a d \bar{\Gamma}
$$

The domain integral presented on right hand side of Eq. (2), which includes stress derivatives, must also be treated. This integral term can be transformed into integrals written on boundaries $\bar{\Gamma}_{1}^{C}$ and $\bar{\Gamma}_{2}^{C}$. In this regard, the width of the 
initial stress region is assumed as thin enough if compared to its length. Therefore, the stress' variation along direction $\bar{x}_{1}$ is null. Therefore

$$
\sigma_{j k, k}^{0}=\frac{\partial \sigma_{j k}^{0}}{\partial x_{k}}=\frac{\partial \sigma_{j k}^{0}}{\partial \bar{x}_{m}} \frac{\partial \bar{x}_{m}}{\partial x_{k}}=\frac{\partial \sigma_{j k}^{0}}{\partial \bar{x}_{2}} \frac{\partial \bar{x}_{2}}{\partial x_{k}}=\frac{\partial \sigma_{j k}^{0}}{\partial \bar{x}_{2}} t_{k}=\frac{\partial\left(\sigma_{j k}^{0} t_{k}\right)}{\partial \bar{x}_{2}}
$$

in which $t_{k}$ contains the outward normal components along $\bar{x}_{2}$. Based on these assumptions, the domain integral presented on right hand side of Eq. (2) can be rewritten as

$$
-\int_{\Omega_{0}} u_{l j}^{*} \sigma_{j k, k}^{0} d \Omega_{0}=-\int_{\Omega_{0}} u_{l j}^{*} \frac{\partial\left(\sigma_{j k}^{0} t_{k}\right)}{\partial \bar{x}_{2}} d \Omega_{0}=-\int_{\bar{x}_{2}} u_{l j}^{*} \frac{\partial\left(\sigma_{j k}^{0} t_{k}\right)}{\partial \bar{x}_{2}} 2 a d \overline{x_{2}}
$$

Equation (8) can be integrated over direction $\bar{x}_{2}$ leading to

$$
-\int_{\bar{x}_{2}} u_{l j}^{*} \frac{\partial\left(\sigma_{j k}^{0} t_{k}\right)}{\partial \bar{x}_{2}} 2 a d \bar{x}_{2}=-\left[u_{l j}^{*}\left(\sigma_{j k}^{0} t_{k}\right) 2 a\right]_{\bar{x}_{2}^{2}}^{\bar{x}_{2}}+\int_{\bar{x}_{2}} \frac{\partial u_{l j}^{*}}{\partial \bar{x}_{2}}\left(\sigma_{j k}^{0} t_{k}\right) 2 a d \bar{x}_{2}
$$

As the width of the initial stress zone is small, the first term on right hand side of Eq. (9) becomes null. Considering that $d \bar{x}_{2}^{C}=d \bar{\Gamma}$ and $\sigma_{j k}^{0} t_{k}=p_{j}^{02}$, Eq. (9) can be rewritten as

$$
-\int_{\Omega_{0}} u_{l j}^{*} \sigma_{j k, k}^{0} d \Omega_{0}=\int_{\bar{\Gamma}} \frac{\partial u_{l j}^{*}}{\partial \bar{x}_{2}} p_{j}^{02} 2 a d \bar{\Gamma}
$$

where $p_{j}^{02}$ indicates the tractions aligned to direction $\bar{x}_{2}$.

Therefore, based on results presented in Eq. (6) and Eq. (10), the domain integral shown in Eq. (1) can be transformed as

$$
\int_{\Omega_{0}} \sigma_{j k}^{0} \varepsilon_{l j k}^{*} d \Omega_{0}=\int_{\bar{\Gamma}} \frac{\partial u_{l j}^{*}(f, S)}{\partial \bar{x}_{1}} p_{j}^{01}(S) 2 a d \bar{\Gamma}+\int_{\bar{\Gamma}} \frac{\partial u_{l j}^{*}}{\partial \bar{x}_{2}} p_{j}^{02} 2 a d \bar{\Gamma}=\int_{\bar{\Gamma}} \frac{\partial u_{l j}^{*}}{\partial \bar{x}_{k}} p_{j}^{0 k} 2 a d \bar{\Gamma}
$$

In order to avoid the local aspect of this deduction, Eq. (11) can be finally rewritten as

$$
\int_{\Omega_{0}} \sigma_{j k}^{0} \varepsilon_{l j k}^{*} d \Omega_{0}=\int_{\Gamma} \frac{\partial u_{l j}^{*}}{\partial \bar{x}_{k}} p_{j}^{0 k} 2 a d \bar{\Gamma}=\int_{\Gamma} \frac{\partial u_{l j}^{*}}{\partial x_{m}} \frac{\partial x_{m}}{\partial \bar{x}_{k}} p_{j}^{0 k} 2 a d \bar{\Gamma}
$$

Until now, a domain integral defined over any thin zone was transformed into a line integral. Based on this formulation, stress and displacement analyses for domains where nonlinear behaviours are assumed inside particular narrow regions can be performed. For instance, crack growth analysis in which cohesive stresses are assumed over a finite strip.

Although a numerical algorithm based on the definition of a thin finite process zone could be derived, as shown above, the nonlinear zone must be wide enough to guarantee initial stress finite values. When the thickness goes to zero, infinite initial stresses are required due to the nature of the problem. Thus, in order to write a proper integral term for which the strip thickness is assumed as zero, a new tensor, denominated dipole, must be defined. Therefore 


$$
\frac{\partial x_{m}}{\partial \bar{x}_{k}} p_{j}^{0 k} 2 a=q_{j}^{m}
$$

This new variable is given by finite values when the initial stress goes to infinity. Then, considering this new variable and the discussion presented above, Eq. (1) can be redefined as

$$
c_{l k}^{i} u_{k}^{i}+\int_{\Gamma} p_{l k}^{*} u_{k} d \Gamma=\int_{\Gamma} u_{l k}^{*} p_{k} d \Gamma+\int_{\Gamma} G_{m}^{l j} q_{j}^{m} d \bar{\Gamma}
$$

where $G_{m}^{l j}=\partial u_{l j}^{*} / \partial x_{m}$. This new kernel is defined by

$$
G_{m}^{l j}=u_{l j, m}^{*}=\frac{-1}{8 \pi G(1-v) r}\left\{(3-4 v) r_{, m} \delta_{l j}-r_{, j} \delta_{l m}-r_{, l} \delta_{j m}+2 r_{, j} r_{l} r_{m}\right\}
$$

In order to derive the stress integral representation, a similar development can be followed. In this regard, Eq. (14) has to be derived and the Hooke's law applied. This procedure leads to

$$
\sigma_{i m}+\int_{\Gamma} S_{\text {ink }}(f, S) u_{k}(S) d \Gamma=\int_{\Gamma} D_{i m k}(f, S) p_{k}(S) d \Gamma+\int_{\Gamma} G_{i m}^{l i}(f, S) q_{j}^{l}(S) d \bar{\Gamma}+g_{i m}^{l i}\left(\sigma_{l j}(p)\right)
$$

in which $g_{i m}^{l j}\left(\sigma_{l j}(p)\right)$ is a free term that appears due to the singularity of the problem. The kernel $G_{i m}^{l j}$ is given by

$$
G_{i m}^{l j}=\frac{1}{4 \pi(1-v) r^{2}}\left\{\begin{array}{l}
(1-2 v)\left(\delta_{i j} \delta_{l m}+\delta_{j m} \delta_{i l}-\delta_{j l} \delta_{i m}\right)-2(1-2 v)\left(r_{, m} r_{l} \delta_{i j}+r_{i, l} r_{l} \delta_{m j}-r_{, j} r_{, l} \delta_{i m}\right)+ \\
+2\left(r_{, j} r_{m} \delta_{i l}+r_{, j} r_{i} \delta_{m l}+r_{, m} r_{i} \delta_{j l}\right)-8 r_{, i} r_{, j} r_{, m} r_{, l}
\end{array}\right\}
$$

Other parameters widely important for modelling fracture mechanics problems are the crack opening displacements. Regarding the proposed formulation, it can be obtained from Eq. (14), which has to be applied for collocation points taken over the dipole line, where discontinuities of displacements and stresses will appear. This equation has to be written for points symmetrically positioned at $S^{+}$and $S^{-}$, as indicated in Fig. (1). As a result, different displacement representations are achieved for these points. By subtracting them, the crack opening displacements are obtained as follows

$$
\left\{\begin{array}{l}
\Delta w_{1} \\
\Delta w_{2}
\end{array}\right\}=\left[\begin{array}{cc}
\frac{1-2 v}{2 G(1-v)} & 0 \\
0 & \frac{1}{G}
\end{array}\right]\left\{\begin{array}{l}
q_{1}^{1} \\
q_{2}^{1}
\end{array}\right\}
$$

It is worth to stress that the presence of dipoles lead to a displacement discontinuity. In Eq. (18), $\Delta w_{1}$ represents the crack opening displacement (mode I) and $\Delta w_{2}$ the crack sliding displacement (mode II).

\section{Algebraic equations}

In previous section, the integral representations with additional terms for modelling stress and displacement discontinuities were derived. The boundary discretization leads to the known matrices $\mathrm{H}$ and $\mathrm{G}$, which take into account boundary displacements and tractions. The remaining term can also be written 
into its algebraic form if the variable $q_{j}^{m}$ is assumed to be approximate by standard shape functions along the crack line. After discretizing the whole crack path by elements and performing properly the integrals over them, Eq. (14) and Eq. (16) assume the following algebraic representations

$$
\begin{gathered}
H U=G P+K Q \\
\sigma+H^{\prime} U=G^{\prime} P+K^{\prime} Q
\end{gathered}
$$

The boundary conditions can be applied in Eq. (19). As usual in BEM formulations, all unknown boundary values are stored in a vector $X$ and all known in a vector $F$. Therefore

$$
A X=B F+K Q \Rightarrow X=M+R Q
$$

where matrices $\mathrm{A}$ and $\mathrm{B}$ result from columns change between matrices $\mathrm{H}$ and $\mathrm{G}$. $\mathrm{M}$ and $\mathrm{R}$ are defined as $M=A^{-1} B F$ and $R=A^{-1} K$.

Similarly, the boundary conditions can be applied in Eq. (20). Performing this step together with the result presented by Eq. (21) one obtains

$$
\sigma+A^{\prime} X=B^{\prime} F+K^{\prime} Q \Rightarrow \sigma=N+S Q
$$

in which $A^{\prime}$ and $B^{\prime}$ result from columns change between matrices $H^{\prime}$ and $G^{\prime}$. The terms $\mathrm{N}$ and $\mathrm{S}$ are equal to $N=B^{\prime} F-A^{\prime} M$ and $S=K^{\prime}-A^{\prime} R$, respectively.

The integrals that lead the algebraic equations Eq. (21) and Eq. (22) were evaluated by Gauss-Legendre numerical scheme accomplished with a subelement technique. Based on these procedures, Eq. (14) and Eq.(16) are transformed into algebraic representations with very low integration error. It is worth to mention that due to the singularity present in the kernel $G_{i m}^{l j}$, only discontinuous elements have to be used at the crack path.

\section{Solution technique}

The cohesive crack propagation problem can be solved using Eq. (21) and Eq. (22). The first one leads the solution of boundary values whereas the second equation allows the determination of stresses and displacements at crack surfaces. It is worth to stress that boundary values are determined since dipoles' values in equilibrium configuration are achieved.

The dipoles are calculated in the context of nonlinear solutions, which are performed in incremental form. In this regard, the exceeding stresses are reapplied on the structure and a dipoles variation is determined, as presented in Eq. (22). As a result of this dipoles variation, the crack opening displacements variation is determined (Eq. (18)), which lead a new stress state of equilibrium. This last one is compared with the stresses due to the external loading. The differences between these two stresses' state are reapplied until the residual stresses vector norm be smaller than a specified tolerance, indicating the convergence. This classical procedure is known as constant operator, since all relevant matrices are kept constant during the iterative process. However, this procedure may need too many iterations to achieve the convergence. In order to reduce the amount of required iterations, other numerical procedures can be adopted. The tangent operator is one of them. 
In order to derive the tangent operator for dipoles' formulation, Eq. (22) has to be rewritten into its incremental form. Then

$$
Y\left(\Delta Q_{n}\right)=-\sigma_{n}\left(\Delta Q_{n}\right)+\Delta N_{n}+S \Delta Q_{n}
$$

in which the subscript $\mathrm{n}$ indicates the current load step.

The nonlinear problem is solved considering a Newton-Raphson scheme, then, using prevision and correction steps. In order to determine the corrections required for achieving the equilibrium condition, the equation above has to be represented using a Taylor series. Therefore

$$
Y\left(\Delta Q_{n}^{i+1}\right)=Y\left(\Delta Q_{n}^{i}\right)+\frac{\partial Y\left(\Delta Q_{n}^{i}\right)}{\partial \Delta Q_{n}^{i}} \delta \Delta Q_{n}^{i}+o\left(\delta \Delta Q_{n}^{i}\right)
$$

in which $O$ represents high order terms and the superscript i indicates the current iteration.

At the equilibrium condition, Eq. (24) must be null. In this regard, the equation above can be rewritten for this condition considering only the first two terms of Taylor expansion. Then

$$
Y\left(\Delta Q_{n}^{i}\right)+\frac{\partial Y\left(\Delta Q_{n}^{i}\right)}{\partial \Delta Q_{n}^{i}} \delta \Delta Q_{n}^{i}=0 \Rightarrow \delta \Delta Q_{n}^{i}=-\left[\frac{\partial Y\left(\Delta Q_{n}^{i}\right)}{\partial \Delta Q_{n}^{i}}\right]^{-1} Y\left(\Delta Q_{n}^{i}\right)
$$

Therefore, it is possible to calculate the dipoles' variation based on the stress residuum of the current iteration and on the derivative of $Y$. The term that relates the derivative of $Y$ with respect of $Q$ is denominated tangent operator. It can be explicated as

$$
\frac{\partial Y\left(\Delta Q_{n}^{i}\right)}{\partial \Delta Q_{n}^{i}}=-\frac{\partial\left[\Delta \sigma_{n}^{i}\left(Q_{n}^{i}\right)\right]}{\partial \Delta Q_{n}^{i}}+S
$$

It is worth to mention that $\sigma$ and $Q$ are described on global coordinates, whereas $\Delta w$ is described on local coordinates. Therefore, in order to determine the crack opening displacements, both $\sigma$ and $Q$ must to be described into local coordinates, as shown in Fig. 2.

The transformation of coordinates, global to local and vice-versa, is performed considering the rotation matrix presented below

$$
T=\left[\begin{array}{ccc}
\cos ^{2}(\theta) & \operatorname{sen}(2 \theta) & \operatorname{sen}^{2}(\theta) \\
-\frac{1}{2} \operatorname{sen}(2 \theta) & \cos (2 \theta) & \frac{1}{2} \operatorname{sen}(2 \theta) \\
\operatorname{sen}^{2}(\theta) & -\operatorname{sen}(2 \theta) & \cos ^{2}(\theta)
\end{array}\right]
$$

Thus, the stresses and dipoles can be described into local coordinates as

$$
\begin{aligned}
\Delta \bar{\sigma}_{n}^{i} & =T \Delta \sigma_{n}^{i} \\
\Delta \bar{Q}_{n}^{i} & =T \Delta Q_{n}^{i}
\end{aligned}
$$

where the over bar indicates variables on local coordinate. 


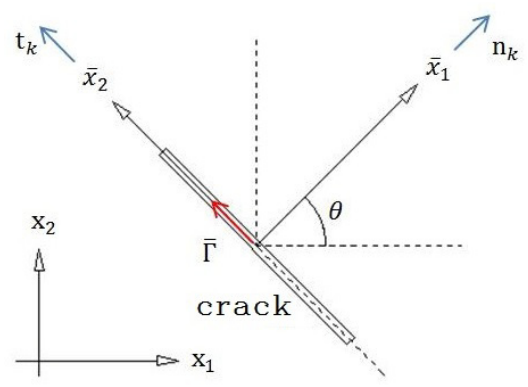

Figure 2: Global and local coordinates.

Therefore, the derivate term positioned at the right hand side of Eq. (26) can be evaluated on local coordinates applying the chain rule as

$$
\frac{\partial \Delta \sigma_{n}^{i}}{\partial \Delta Q_{n}^{i}}=T^{-1} \frac{\partial \Delta \bar{\sigma}_{n}^{i}}{\partial \Delta Q_{n}^{i}} \Rightarrow \frac{\partial \Delta \sigma_{n}^{i}}{\partial \Delta Q_{n}^{i}}=T^{-1} \frac{\partial \Delta \bar{\sigma}_{n}^{i}}{\partial \Delta w_{n}^{i}} \frac{\partial \Delta w_{n}^{i}}{\partial \Delta \bar{Q}_{n}^{i}} \frac{\partial \Delta \bar{Q}_{n}^{i}}{\partial \Delta Q_{n}^{i}}
$$

The first derivate term presented on right hand side of Eq. (29) is obtained according the cohesive criteria adopted, which relates cohesive stresses to crack opening displacements. The second derivate term of this equation is achieved using the result presented in Eq. (18). Then

$$
\frac{\partial \Delta w_{n}^{i}}{\partial \Delta \bar{Q}_{n}^{i}}=\left[\begin{array}{c}
\frac{1-2 v}{2 G(1-v)} \\
0 \\
0
\end{array}\right]
$$

Finally, the last derivate term positioned at the right hand side of Eq. (29) is achieved using the relations presented in Eq. (28). Then

$$
\frac{\partial \Delta \bar{Q}_{n}^{i}}{\partial \Delta Q_{n}^{i}}=T
$$

Therefore, Eq. (29) can be rewritten as

$$
\frac{\partial \Delta \sigma_{n}^{i}}{\partial \Delta Q_{n}^{i}}=T^{-1}\left[\begin{array}{c}
\frac{\partial \Delta \bar{\sigma}_{\text {criteria }}}{\partial \Delta w} \\
0 \\
0
\end{array}\right]\left[\begin{array}{c}
\frac{1-2 v}{2 G(1-v)} \\
0 \\
0
\end{array}\right]^{T} T \Rightarrow \frac{\partial \Delta \sigma_{n}^{i}}{\partial \Delta Q_{n}^{i}}=\frac{1-2 v}{2 G(1-v)} \frac{\partial \Delta \bar{\sigma}_{\text {criteria }}}{\partial \Delta w} R
$$

in which $R$ is given by

$$
R=\left[\begin{array}{ccc}
\cos ^{4}(\theta) & 2 \cos ^{3}(\theta) \operatorname{sen}(\theta) & \cos ^{2}(\theta) \operatorname{sen}^{2}(\theta) \\
\cos ^{3}(\theta) \operatorname{sen}(\theta) & 2 \cos ^{2}(\theta) \operatorname{sen}^{2}(\theta) & \cos (\theta) \operatorname{sen}^{3}(\theta) \\
\cos ^{2}(\theta) \operatorname{sen}^{2}(\theta) & 2 \cos (\theta) \operatorname{sen}^{3}(\theta) & \operatorname{sen}^{4}(\theta)
\end{array}\right]
$$


Finally, based on the results presented in Eq. (32) the tangent operator considering the dipole's formulation assumes the following form

$$
\frac{\partial Y\left(\Delta Q_{n}^{i}\right)}{\partial \Delta Q_{n}^{i}}=S-\frac{1-2 v}{2 G(1-v)} \frac{\partial \Delta \bar{\sigma}_{\text {criteria }}}{\partial \Delta w} R
$$

The tolerance to stop the iterative process within an increment of load is applied on the variation of the crack opening displacement corrections, i.e., $w^{i}-w^{i-1} \leq$ tolerance. Moreover, it is worth to remark that the total crack opening displacement is always required to compute the local tangent operator for the next iteration.

\section{Application}

The three point bending test considered in this example is shown in Fig. 3. The geometry is given by its length, $800 \mathrm{~mm}$, height, $200 \mathrm{~mm}$, and a central notch of $50 \mathrm{~mm}$ deep. The experimental results are shown in [8], from where the following properties were obtained: tensile strength $\sigma_{t}^{c}=3.0 \mathrm{MPa}$, Young's modulus $E=30000 \mathrm{MPa}$, Poisson ratio $v=0.15$ and fracture energy $G_{f}=75 \mathrm{~N} / \mathrm{m}$. In order to model the crack propagation in this structure, three cohesive laws were adopted: linear, bi-linear and exponential. The load was applied into 24 increments and the convergence has been verified with a tolerance of $10^{-5}$, based on the norm of non-equilibrated stress vector.

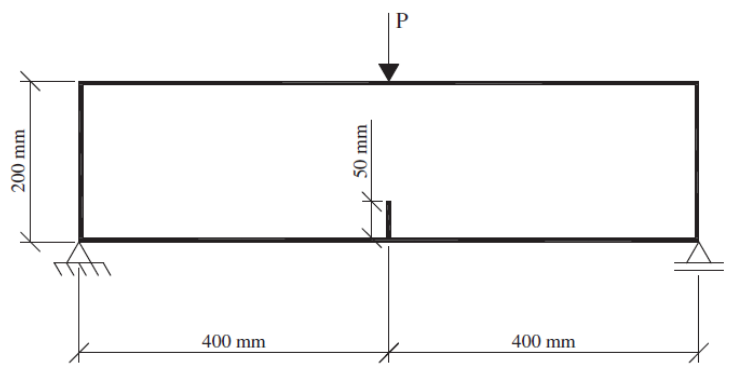

Figure 3: Three point bending specimen.

The load displacement curves achieved in this analysis are shown in Fig. 4, where the results obtained by the proposed formulation are compared with experimental and numerical [4], available in literature. In this figure, the symbol TO indicates the curves constructed using tangent operator.

According this figure, good agreement is observed between experimental results and those achieved by the proposed formulation. Moreover, the results obtained by dipole' formulation are equivalent with those found by dual BEM formulation, indicating its robustness.

The tangent operator leads the use of less iterations to achieve the convergence. Table 1 shows a comparative of amount of iterations required for 


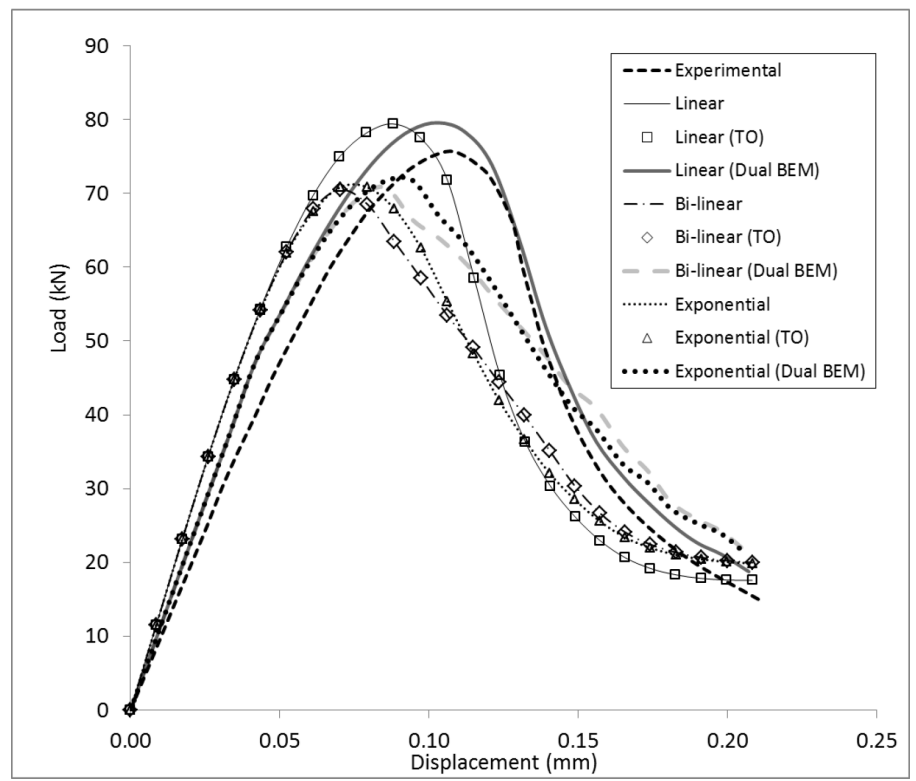

Figure 4: $\quad$ Load $x$ displacement curve.

Table 1: $\quad$ Comparative of iterations.

\begin{tabular}{cccc}
\hline $\begin{array}{c}\text { Cohesive } \\
\text { law }\end{array}$ & Constant operator & Tangent operator & Reduction (\%) \\
\hline Linear & 31002 & 9549 & 69.2 \\
Bi-linear & 154533 & 52085 & 66.3 \\
Exponential & 158136 & 31639 & 80.0 \\
\hline
\end{tabular}

constant and tangent operators for achieving the equilibrium configuration. Regarding this table an excellent performance of tangent operators is observed front the classical nonlinear approach.

\section{Conclusions}

In this paper, the crack growth process in quasi-brittle materials has been studied. This complex structural problem can be modelled solving a nonlinear system of equations, which appears due to the dependency between crack opening displacement and cohesive stresses along the crack path. In order to simulate this nonlinear structural problem, BEM has shown to be an accurate and efficient alternative. An alternative BEM formulation, based on initial stresses field, is proposed in order to simulate the nonlinear effects caused by damaged zones present in narrow regions into the domains. It is worth to stress that the proposed formulation uses three algebraic equations to describe the crack mechanical behaviour whereas the classical dual BEM requires four. 
Two iterative schemes have been applied to solve the nonlinear problem. The first one applies a constant operator, where all relevant matrices are kept constant along the iterative process. The second approach is developed by using a tangent operator. In this case, the derivate set of nonlinear equations is used and the problem is faster solved. The tangent operator has shown to be faster than the constant one. The use of tangent operator has shown to be always recommended to analyze crack propagation problems, particularly for the cases where the after pick region is reached.

\section{Acknowledgement}

Sponsorship of this research project by the São Paulo State Foundation for Research (FAPESP), project number 2011/07771-7 is greatly appreciated.

\section{References}

[1] Barenblatt, G.I. The mathematical theory of equilibrium cracks in brittle fracture. Advances in Applied Mechanics. 7: 55-129, 1962.

[2] Dugdale, D.S. Yielding of steel sheets containing slits. Journal of Mechanics and Physics of Solids. 8:100-104, 1960.

[3] Hillerborg, A.; Modeer, M.; Peterson, P.E. Analysis of crack formation and crack growth in concrete by mean of failure mechanics andfinite elements. Cement Concrete Research. 6:773-782, 1976.

[4] Leonel, E.D.; Venturini, W.S. Non-linear boundary element formulation with tangent operator to analyse crack propagation in quasi-brittle materials. Eng Anal Bound Elem. 34:122-129, 2010.

[5] Moes, N.; Dolbow, J.; Belytschko, T. A finite element method for crack growth without remeshing. Int. J. Numer Meth Engng. 46: 131-150, 1999.

[6] Leonel, E.D.; Venturini, W.S.; Chateauneuf, A. A BEM model applied to failure analysis of multi-fractured structures. Eng Fail Anal.18: 1538-1549, 2011.

[7] Leonel, E.D.; Chateauneuf, A.; Venturini, W.S. Probabilistic crack growth analyses using a boundary element model: applications in linear elastic fracture and fatigue problems. Eng Anal Bound Elem. 36: 944-959, 2012.

[8] Saleh, A.L.; Aliabadi, M.H. Crack-growth analysis in concrete using boundary element method. Eng Fract Mech. 51(4): 533-545, 1995.

[9] Yang, B.; Ravi-Chandar, K. A single-domain dual-boundary-element formulation incorporating a cohesive zone model for elastostatic cracks. Int. J. Frac, 93: 115-144, 1998.

[10] Cen, Z.; Maier, G. Bifurcations and instabilities in fracture of cohesivesoftening structures: A boundary element analysis. Frac of Eng Mat, 1992.

[11] Maier, G.; Novati, G.; Cen, Z. Symmetric Galerkin boundary element method for quasi-brittle-fracture and frictional contact problems. Comp Mech, 13: 74-89, 1993.

[12] Brebbia, C.A.; Dominguez, J. Boundary Elements: An introductory course. Second edition, WIT Press, Southampton, 1992. 